\title{
Nonlinear current-induced forces in Si atomic wires
}

\author{
Zhongqin Yang and Massimiliano Di Ventra* \\ Department of Physics, Virginia Polytechnic Institute and State University, Blacksburg, Virginia 24061
}

(Received 30 January 2003; revised manuscript received 13 March 2003; published 30 April 2003)

\begin{abstract}
We report first-principles calculations of current-induced forces in $\mathrm{Si}$ atomic wires as a function of bias and wire length. We find that these forces are strongly nonlinear as a function of bias due to the competition between the force originating from the scattering states and the force due to bound states. We also find that the shorter the wire, the larger the average force in the wire, suggesting that the wires are more difficult to break under current flow with increasing length. The last finding is in agreement with recent experimental data.
\end{abstract}

DOI: 10.1103/PhysRevB.67.161311

PACS number(s): 73.40.Cg, 73.40.Gk, 85.65.+h

Current-induced atomic motion (electromigration) has always been of concern in microelectronics, since it was found to be a major failure mechanism in aluminum conductors. ${ }^{1}$ Such an effect is more pronounced when the material dimensions are smaller as it is the case, for instance, for metallization lines in conventional circuits. ${ }^{2}$ It does not come as a surprise then that this effect has recently attracted considerable interest in nanoscience. In particular, current-induced forces in atomic ${ }^{3-7}$ and molecular wires ${ }^{8,9}$ have been investigated. These systems show interesting physical properties, which make them promising candidates for future electronic applications. ${ }^{10}$ It was found that current-induced forces in a nanoscale wire can distort its local atomic structure and affect its stability according to the external bias and the wire length. ${ }^{4-7,9,11}$ Longer atomic wires, for instance, have been found to be more stable with respect to current-induced forces than shorter wires. ${ }^{12}$

Over the years, considerable theoretical and experimental works have been devoted to understand the microscopic origin of electromigration. ${ }^{13}$ It has become clear, for instance, that this phenomenon depends strongly on the microscopic details of the self-consistent electric field that is created upon scattering of the electrons across the region of interest. Despite the progress in the fundamental understanding of current-induced forces, however, it is still unclear what determines, e.g., the resistance of atomic and molecular wires to electromigration effects or what is the degree of dependence of these forces on the external bias and wire length.

In this Rapid Communication, we explore the above issues using the first-principles approaches. In particular, we study current-induced forces as a function of bias in $\mathrm{Si}$ atomic wires of varying length. There have been several experimental realizations of $\mathrm{Si}$ wires ${ }^{14}$ and the interest in the nanoscale properties of $\mathrm{Si}$ stems from the fact that it is the material of choice in microelectronics. We find that currentinduced forces are strongly nonlinear as a function of bias due to the competition between the force originating from the continuum of states and the force due to states in the discrete part of the spectrum. The force originating from the bare electrodes (we will call this force, direct ${ }^{15}$ ) varies almost linearly as a function of bias. We also find that shorter the wire the larger the average force in the wire suggesting that wires of increasing length are more difficult to break under current flow. ${ }^{16}$ The last finding is in agreement with recent experimental data. ${ }^{12}$
A schematic diagram of the system investigated is depicted in the inset of Fig. 1. It consists of a $\mathrm{Si}$ atomic wire sandwiched between two gold surfaces that we model with ideal metals (jellium model). ${ }^{17,18}$ The interior electron density of the electrodes is taken equal to the value for metallic gold $\left(r_{s} \approx 3\right)$. The electric current is calculated using the method described in Refs. 17,18. The electron wave functions are computed by solving the Lippman-Schwinger equation iteratively to self-consistency in a steady state. Exchange and correlation are included in the density-functional formalism within the local-density approximation. ${ }^{17,18}$ The current is computed from the wave functions $|\psi\rangle$ of the electrode-molecule system. The force $\mathbf{F}$ acting on a given atom at position $\mathbf{R}$ due to the electron distribution as modified by the external bias is given by the Hellmann-Feynman type of theorem developed in Ref. 9: ${ }^{19}$

$$
\mathbf{F}=-\sum_{i}\left\langle\psi_{i}\left|\frac{\partial H}{\partial \mathbf{R}}\right| \psi_{i}\right\rangle-\lim _{\Delta \rightarrow 0} \int_{\sigma} d E\left\langle\psi_{\Delta}\left|\frac{\partial H}{\partial \mathbf{R}}\right| \psi_{\Delta}\right\rangle .
$$

The sum and integral in Eq. (1) include spin variables also. The first term on the right-hand side(RHS) of Eq. (1) is similar to the usual Hellmann-Feynman contribution to the force due to localized electronic states $\left|\psi_{i}\right\rangle$. The second term is the contribution to the force due to the continuum of states. ${ }^{9}$ It is calculated by constructing, for each energy in the continuum, square-integrable wave functions $\left|\psi_{\Delta}\right\rangle$ in an energy region $\Delta$

$$
\left|\psi_{\Delta}\right\rangle=\mathcal{A} \int_{\Delta} d E \psi
$$

where $\mathcal{A}$ is a normalization constant and the $\psi$ 's are singleparticle wave functions in the continuum, solutions of the Lippmann-Schwinger equation. ${ }^{9}$ The continuum integration $\sigma$ covers the part of the spectrum occupied by the electrons at a given bias. Finally, the total force on the atom includes a trivial ion-ion interaction.

We start the calculations by first relaxing the atomic positions at zero bias. For all different wire lengths, the relaxed Si-jellium surface bond length is about 2 a.u. and the relaxed $\mathrm{Si}-\mathrm{Si}$ bond distance is about 4.2 a.u. In Figs. 1(a)-1(c) we plot the total force as a function of bias on each $\mathrm{Si}$ atom for wires composed of two, three, and four Si atoms, respectively. The atomic positions have been fixed at the equilibrium position at zero bias, and the atoms are labeled with 


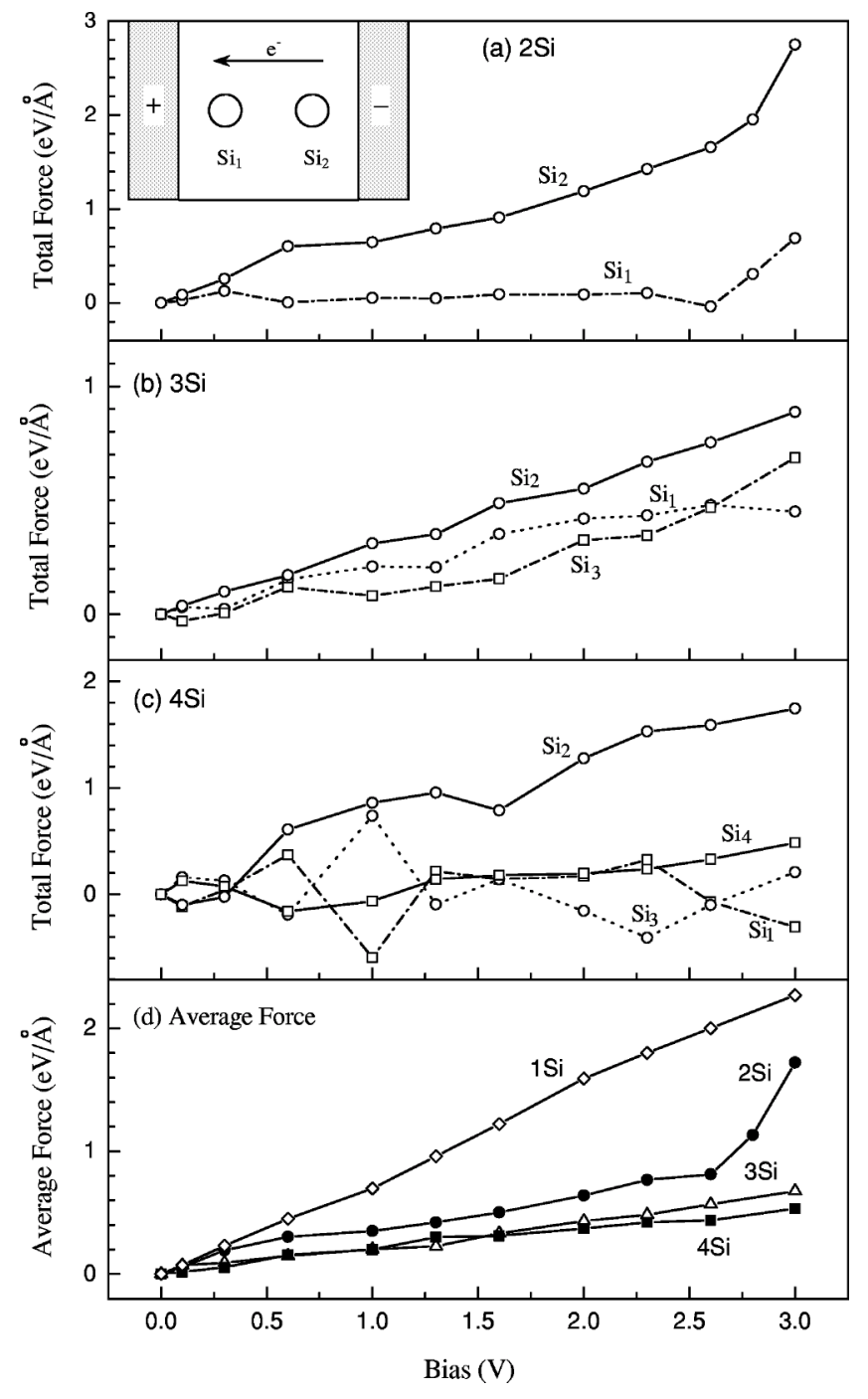

FIG. 1. Total force as a function of bias in atomic wires containing (a) two, (b) three, and (c) four Si atoms. The inset shows a schematic of one of the wires investigated. The atoms are labeled with increasing number from the left electrode (see inset). (d) The average force in the $\mathrm{Si}$ wires. The left electrode is positively biased. Positive force pushes the atom opposite to electron flow.

increasing number, starting from the closer to the left electrode (see Fig. 1). In Fig. 1(d), we plot the average force (sum of the forces on each atom divided by the number of atoms in the wire) as a function of bias and the force for a wire composed of a single $\mathrm{Si}$ atom. Positive force pushes the atom to the right, i.e., opposite to electron flow (the left electrode is positively biased). It is clear from Fig. 1(a)-1(c) that the force on atoms is a nonlinear function of the bias [except for the single-atom wire and $\mathrm{Si}_{2}$ in Fig. 1(b), see below and Ref. 20] while the average force, Fig. 1(d), saturates with the number of atoms in the wire. We first discuss the nonlinear behavior of the forces and then discuss the average force.

For a small external bias $(0.01 \mathrm{~V}$, i.e., linear-response regime), the current-induced forces in the 3-Si wire satisfy the zero-force sum rule, while for the 2-Si and 4-Si wires all atoms are pushed opposite to the current flow with almost equal force as it can be inferred from symmetry considerations $^{7}$ (not shown in Fig. 1). On the other hand, strong nonlinearities in the current-induced forces appear at voltages above $0.1 \mathrm{~V}$. For instance, in the 2-Si wire the $\mathrm{Si}$ atom closer to the left electrode ( $\mathrm{Si}_{1}$ in the inset of Fig. 1) is pushed against the electron flow for biases less than $0.5 \mathrm{~V}$ and moves along the electron flow for larger biases. On the other hand, the second $\mathrm{Si}$ atom $\left(\mathrm{Si}_{2}\right.$ in the inset of Fig. 1) is pushed against the electron flow at all external voltages. Similar effects, involving different atoms in the wire, occur at even smaller voltages for the 4-Si wire. In the same vein, for the 3-Si wire the zero-force sum rule is not satisfied already at $0.1 \mathrm{~V}$. It is interesting to note that for large biases, the largest force occurs on the second $\mathrm{Si}$ atom from the left. The break up of the wires is thus likely to nucleate from the bonds of this atom. In general, each atom experiences a force due to current flow that is nonlinear in the external bias. Such nonlinearities affect the atomic redistribution in the wire and eventually its resistance to current-induced rupture. In order to understand this nonlinear behavior, we study the following different contributions to the forces as a function of bias: (a) the contribution from the bulk electrodes electrostatic potential (direct force ${ }^{13}$ ), (b) the contribution from the continuum part of the spectrum, and (c) the part of the force originating from the discrete spectrum. The force originating from the ion-ion interaction does not depend on the bias and we do not discuss it here. For the sake of simplicity, we discuss the 3-Si wire case. Similar considerations are valid for other wire lengths. We plot in Fig. 2 the three different contributions considered. As expected, the direct force is almost linear with the bias. Small deviations from linearity appear due to the small deviations from linear decay of the electrostatic potential close to the electrode surfaces. ${ }^{17,18}$ Furthermore, due to this deviation, the force on the central atom in the wire is larger than the force on the two atoms close to the electrode surfaces at any bias (see Fig. 2). The latter atoms also experience a force of similar magnitude with deviations occurring at large voltages. From Fig. 2 it is also evident that for biases larger than $0.5 \mathrm{~V}$, the major contribution to the force on each atom comes from the direct force. The nonlinearities in the total force then originate from the competition between the force due to the states in the continuum (scattering states) and the force due to the bound states (in this case, those states below the band bottom of the left electrode). The force on the central atom of the wire $\left(\mathrm{Si}_{2}\right)$ due to the scattering states pushes the atom to the left, i.e., along with the electron flow. This force is almost linear with the bias. The force on the same atom due to the discrete spectrum, on the other hand, is almost zero even at very large voltages. This behavior can be understood by looking at the extra charge localized on the atom at any given bias. This quantity can be estimated by integrating the charge around a given atom in a box centered on that atom with one face parallel to the electrode surfaces and whose size in the perpendicular direction is equal to the bond distance between the atoms. The difference between this charge at zero bias and the corresponding charge at any given bias is plotted in Fig. 3 for three different Si atoms. Apart from small fluctuations as a function of bias, the extra charge on the central 


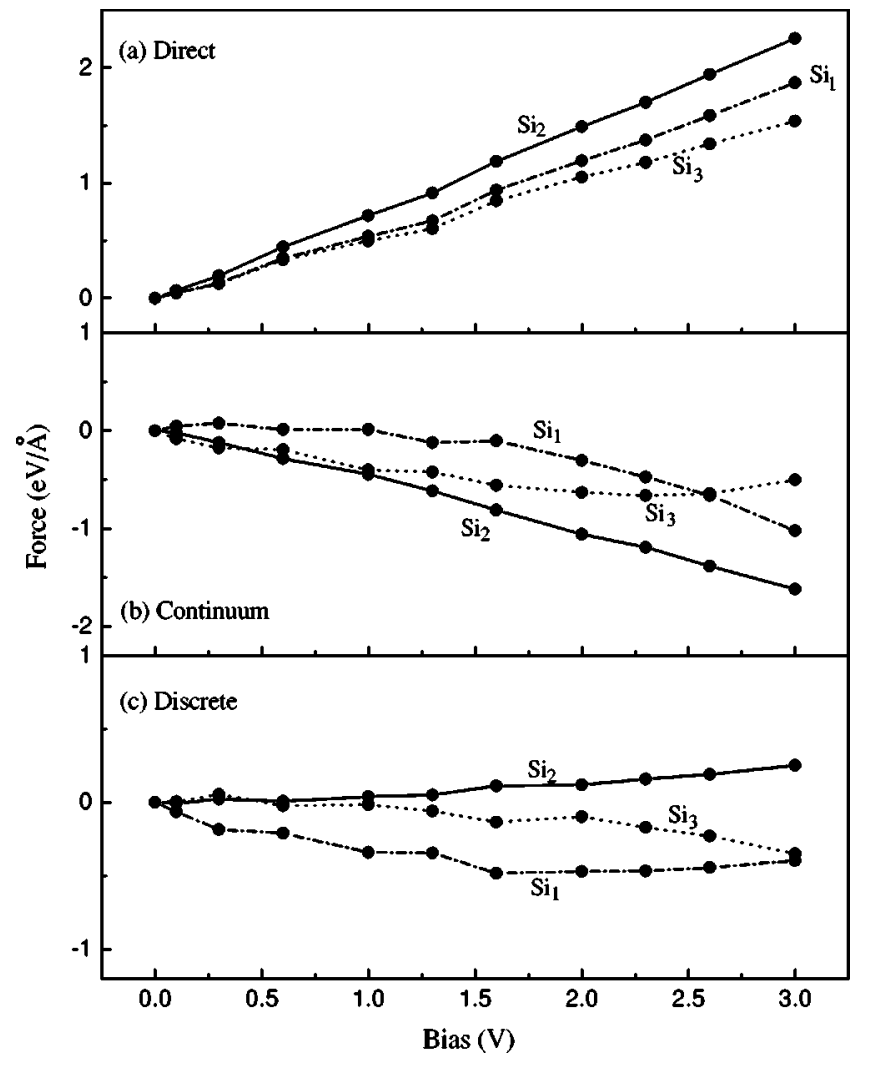

FIG. 2. Different contributions to the total force for a 3-Si wire: (a) direct force (see text), (b) scattering-state contribution, and (c) contribution from the discrete spectrum.

atom is zero. The charge on this atom is thus practically constant at any bias. Since, in this system, the number of bound states does not change with bias, their contribution to the force is constant [see Fig. 2(c)]. On the other hand, the force due to the continuum [second term on the RHS of Eq. (1)] increases linearly with bias due to the larger energy integration in Eq. (1) for larger voltages. ${ }^{20}$

The continuum and discrete contributions to the force are nonlinear for the atoms closer to the electrodes $\left(\mathrm{Si}_{1}\right.$ and $\mathrm{Si}_{3}$ of Fig. 2). For the $\mathrm{Si}_{1}$ atom, for instance, the continuum force

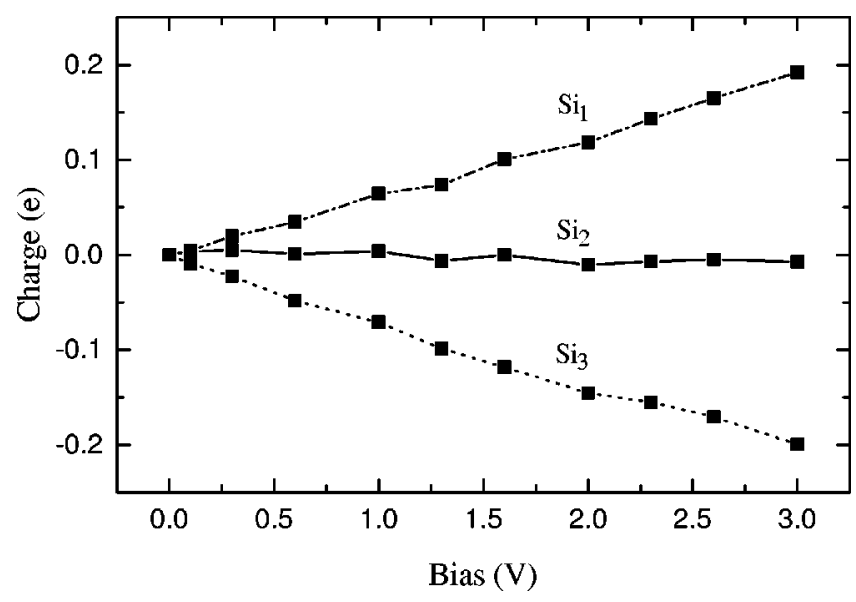

FIG. 3. Extra charge (see text) on the three $\mathrm{Si}$ atoms in the $3-\mathrm{Si}$ wire as a function of bias.

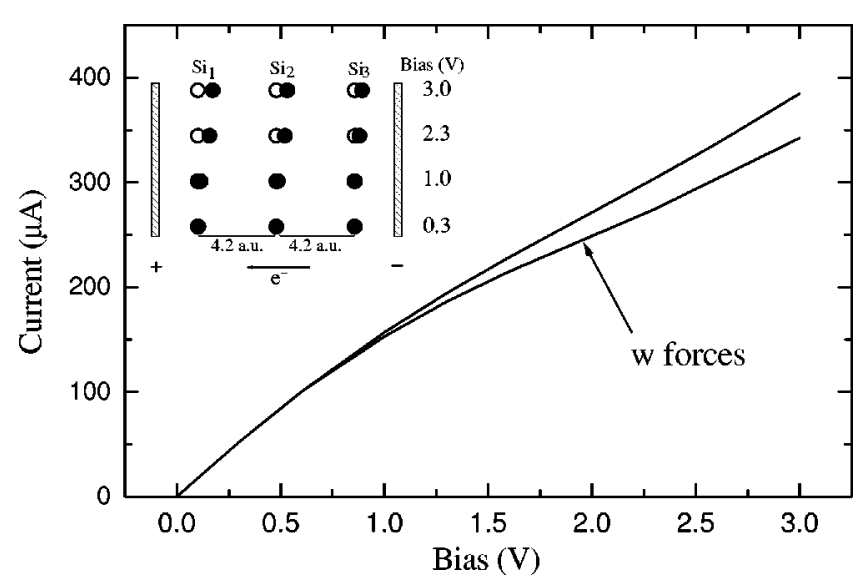

FIG. 4. I-V curve of the 3-Si wire with and without the effect of current-induced atomic relaxations. The inset shows the unrelaxed (open circles) and relaxed (full circles) atomic positions of the $\mathrm{Si}$ atoms between the two electrodes (vertical thin rectangles) at selected biases. The atoms move opposite to the electron flow.

is almost constant up to about $1 \mathrm{~V}$, while the force from the discrete spectrum increases in magnitude with bias in the same voltage range (the sign of the force corresponds to the atom pushed along with the current flow). For biases above 1 $\mathrm{V}$, the continuum force increases in magnitude and the force due to bound states is almost constant. An opposite trend is observed for the $\mathrm{Si}_{3}$ atom (see Fig. 2). The opposite trend can be explained again by looking at the extra charge on these atoms (Fig. 3). This charge is of similar magnitude but of opposite sign indicating that there is a charge transfer from the right electrode to the left electrode when current flows with consequent creation of a local electric dipole. ${ }^{21}$

While it is difficult to extract trends in the overall resistance of the $\mathrm{Si}$ wires to electromigration by looking at the forces on each atom, the average force follows quite a simple trend as a function of bias and wire length. In Fig. 1(d), we plot such a quantity for wires of two, three, and four $\mathrm{Si}$ atoms. For a comparison, we also plot the force for a single $\mathrm{Si}$ atom. The average force is almost linear as a function of bias even for large biases (deviations occur above $2.5 \mathrm{~V}$ for the 2-Si wire). Furthermore, the smaller the wire length the larger the force and, in particular, it is almost equal for the 3and 4-Si wires. ${ }^{22}$ Incidentally, the average current per atom follows a similar trend. This is consistent with the fact that the wire has no defects and the resistance is mainly determined by the contacts. The saturation of the force with increasing length can be understood as follows. By increasing the wire length, the direct force will eventually be zero as the boundary effects will be less important with increasing separation of the two leads. On the other hand, the force due to the charge redistribution in the wire under current flow will reach a constant value on average, since the wave functions of the wire will resemble states that are evenly distributed across the length of the wire. Similar trends have been observed in experiments with $\mathrm{Au}$ atomic wires. ${ }^{12}$

We conclude this paper by studying the effects of currentinduced atomic relaxations on the current-voltage (I-V) characteristics of $\mathrm{Si}$ wires. It was found by Di Ventra et al. ${ }^{9,11}$ that current-induced atomic relaxations in molecular wires 
do not substantially affect the absolute value of the current for large voltages and current densities. We find that this is also valid in the present case. This is illustrated in Fig. 4, where we plot the I-V characteristics for the 3-Si wire with and without current-induced atomic relaxations. In the inset of the same figure, we show the relaxed atomic positions for each atom at selected biases. It is clear from Fig. 4 that small changes in the absolute value of the current, when the atoms are relaxed, are observed even at large biases. Similar results are valid for the other $\mathrm{Si}$ wires as well.

In conclusion, we have reported first-principles calculations of current-induced forces in $\mathrm{Si}$ atomic wires. We find that these forces are strongly nonlinear as a function of bias. Since the direct force from the bare electrodes is almost linear as a function of bias, the nonlinearity originates from the competition between the scattering-state and discretespectrum force. We have also found that the shorter the wire the larger the force, suggesting the wires are more difficult to break under current flow with increasing length. Finally, current-induced relaxations are found to change only slightly the absolute value of the current even at large voltages.

We thank Y.-C. Chen for useful discussions. This work was supported in part by the National Science Foundation Grants Nos. DMR-01-02277 and DMR-01-33075, Carilion Biomedical Institute, and Oak Ridge Associated Universities. Acknowledgment is also made to the Donors of The Petroleum Research Fund, administered by the American Chemical Society, for partial support of this research. The calculations reported in this paper were performed on the beowulf cluster of the Laboratory for Advanced Scientific Computing and Applications at Virginia Tech.
*Electronic address: diventra@vt.edu

${ }^{1}$ See, e.g., I.A. Blech and H. Sello, in Physics of Failure in Electronics, edited by T.S. Shilliday and J. Vaccaro (USAF, Rome Air Development Center, Rome, 1967), Vol. 5, p. 496.

${ }^{2}$ M. Hauder, J. Gstottner, W. Hansch, and D. Schmitt-Landsiedel, Appl. Phys. Lett. 78, 838 (2001).

${ }^{3}$ N.D. Lang, Phys. Rev. B 45, 13599 (1992); 49, 2067 (1994).

${ }^{4}$ N. Agraï, C. Untiedt, G. Rubio-Bollinger, and S. Vieira, Phys. Rev. Lett. 88, 216803 (2002).

${ }^{5}$ K. Itakura, K. Yuki, S. Kurokawa, H. Yasuda, and A. Sakai, Phys. Rev. B 60, 11163 (1999).

${ }^{6}$ H. Yasuda and A. Sakai, Phys. Rev. B 56, 1069 (1997).

${ }^{7}$ T.N. Todorov, J. Hoekstra, and A.P. Sutton, Philos. Mag. B 80, 421 (2000); T.N. Todorov, J. Hoekstra, and A.P. Sutton, Phys. Rev. Lett. 86, 3606 (2001).

${ }^{8}$ B.Q. Wei, R. Vajtai, and P.M. Ajayan, Appl. Phys. Lett. 79, 1172 (2001).

${ }^{9}$ M. Di Ventra and S.T. Pantelides, Phys. Rev. B 61, 16207 (2000); M. Di Ventra, S.T. Pantelides, and N.D. Lang, Phys. Rev. Lett. 88, 046801 (2002).

${ }^{10}$ See, e.g., Molecular Electronics II, edited by A. Aviram, M. Ratner, and V. Mujica (New York Academy of Sciences, New York, 2002).

${ }^{11}$ M. Di Ventra, N.D. Lang, and S.T. Pantelides, Chem. Phys. 281, 189 (2002).

${ }^{12}$ A.I. Yanson, G. Rubio-Bollinger, H.E. van den Brom, N. Agraï, and J.M. van Ruitenbeek, Nature (London) 395, 783 (1998).

${ }^{13}$ R.S. Sorbello, Solid State Physics, edited by H. Ehrenreich and F. Spaepen (Academic, New York, 1998), Vol. 51, p. 159, and references therein; R. Landauer and J.W.F. Woo, Phys. Rev. B 10, 1266 (1974); A.K. Das and R. Peierls, J. Phys. C 8, 3348 (1975); L.J. Sham, Phys. Rev. B 12, 3142 (1975).

${ }^{14}$ See, e.g., Y. Cui, Z. Zhong, D. Wang, W. Wang, and C. M. Lieber, Nano Lett. 3, 149 (2003); Y. Huang, X. Duan, Y. Cui, L.J. Lauhon, K. Kim, and C.M. Lieber, Science (Washington, DC, U.S.) 294, 1313 (2001).

${ }^{15}$ Note that this definition of direct force is not unique in literature (see, e.g., Ref. 13). Furthermore, the part of the "electron wind force" defined in the literature (see Ref. 13) as due to the energy transfer from the electrons to the ions is estimated to be very small in the present case. For instance, for a 3-Si wire and a bias of $0.1 \mathrm{~V}$ the electric current is about $17 \mu \mathrm{A}$. Assuming a typical mean-free path of $1000 \AA$ for ballistic conduction, we obtain a collision time of $0.5 \times 10^{-13} \mathrm{~s}$ at the gold Fermi velocity. During this collision time there are, on average, about five collisions due to current flow. An upper bound of the force per collision is the change of momentum due to an electron at the right Fermi level that scatters to the left Fermi level. The total force is then the sum of this force over all collisions, i.e., $\sim 0.005 \mathrm{eV} / \AA$, which is one order of magnitude smaller than the force due to the redistribution of charge (see Fig. 1). This estimate increases with the square root of the bias and is thus one to two orders of magnitude smaller than the force we discuss in the text.

${ }^{16}$ We neglect local heating effects that might contribute to mechanical deformations of the wires. See, e.g., Y.-C. Chen, M. Zwolak, and M. Di Ventra, cond-mat/0302425 (unpublished).

${ }^{17}$ N.D. Lang, Phys. Rev. B 52, 5335 (1995); 49, 2067 (1994); Z. Yang, A. Tackett, and M. Di Ventra, Phys. Rev. B 66, 041405 (2002).

${ }^{18}$ M. Di Ventra and N.D. Lang, Phys. Rev. B 65, 045402 (2002).

${ }^{19}$ Note that a misprint appears in the expression of the force in Refs. 9,18,11. A negative sign is missing in the definition of forces in these references. See, M. Di Ventra, S.T. Pantelides, and N.D. Lang, Phys. Rev. Lett. 89, 139902 (2002).

${ }^{20}$ Similar considerations explain the almost linear dependence of the force on the single-atom wire [see Fig. 1(d)]. In this case, the extra charge on the atom is almost zero at any bias with consequent constant contribution from the discrete spectrum, and linear contribution from the continuum spectrum as a function of bias.

${ }^{21}$ It is interesting to note, at this point, that the sum of the continuum-and discrete-state contributions to the force is larger for the second atom from the right electrode at all biases. A similar result has been inferred by Brandbyge, et al., [M. Brandbyge, N. Kobayashi, and M. Tsukada, Phys. Rev. B 60, 17064 (1999)] from the analysis of the voltage drop in gold wires, suggesting that this effect is material independent.

${ }^{22}$ Using the tight-binding approach, T. N. Todorov, J. Hoekstra, and A. P. Sutton, have shown that in linear response the largest current-induced force is almost constant for wire lengths of three atoms or more (Ref. 7). We show here that this trend is also valid for the average force of $\mathrm{Si}$ wires at any bias. 